\title{
Los cuerpos enseñantes en el aula de filosofía*
}

\author{
Janett Tourn Travers \\ Instituto de Profesores Artigas (IPA- CFE), Montevideo, Uruguay \\ jantourn@gmail.com
}

\begin{abstract}
No somos de esos que solo llegan a tener ideas entre libros, por impulso de libros: estamos acostumbrados a pensar al aire libre, andando, subiendo, bailando, y donde más nos gusta hacerlo es en montañas solitarias o justo al lado del mar, allí donde incluso los caminos se hacen relfexivos.
\end{abstract}

(Nietzsche, 1990, p. 387)

\section{RESUMEN}

Loque siguecorrespondealosprincipales resultados de la investigación realizada desde un abordaje etnográfico a cinco estudiantes de profesorado de Filosofía* del Instituto de Profesores Artigas (IPA) de la ciudad de Montevideo, Uruguay. Se enmarca en el campo de la didáctica de la filosofía, en la que se articulan dos campos de saberes: los estudios sobre el cuerpo (fundamentalmente desde la filosofía y la antropología del cuerpo) y las producciones en torno a la enseñanza de la filosofía. El trabajo de campo fue realizado entre los años 2013 y 2014, el procesamiento de la información se realizó fundamentalmente durante el año $2015 \mathrm{y}$ fue defendida en el año 2016. La investigación se desarrolla como estudio de caso, desde un abordaje cualitativo, de corte interpretativista y etnográfico. La relevancia de la misma está dada a partir de la necesidad de incorporar el ámbito corporal al campo de la formación docente y específicamente a la formación de docentes de filosofía. El ámbito corporal se entiende como un aspecto constituyente de la subjetividad y, por ende, de la forma de existir y presentarse de los sujetos en el mundo.

La finalidad de la investigación, si bien se enmarca como estudio exploratorio por no contar con antecedentes directos sobre el campo a indagar, refiere a la necesidad de integrar el trabajo del sujeto docente consigo mismo, con su propia corporeidad, en tanto cuerpos afectantes y afectados en el marco del aula filosófica.

Palabras clave: didáctica de la filosofía; cuerpos; estudios sobre el cuerpo; cuerpos enseñantes.

Cómo citar: Tourn Travers, J. (2020). Los cuerpos enseñantes en el aula de Filosofía. Ciencias Sociales y Educación, 9(17), 95-115. https://doi.org/10.22395/csye.v9n17a5

Recibido: 12 de noviembre de 2019.

Aprobado: 11 de febrero de 2020.

** Esta formación refiere a formación de grado cuyo título es profesor de Enseñanza Media con especialidad en Filosofía, expedido por el Consejo de Formación en Educación de la Administración Nacional de Educación Pública de Uruguay. La formación refiere a formar docentes destinados a trabajar fundamentalmente en la enseñanza media, lo que tradicionalmente en Uruguay se designan como liceos. 


\section{The Teaching Bodies in the Philosophy Classroom}

\section{ABSTRACT}

The following texts correspond to the main findings from research performed through an ethnographic approach to five professoriate students in Philosophy ${ }^{*}$ from the Artigas Professors Institute (IPA in Spanish) in the city of Montevideo, Uruguay. It is framed within the field of didactics in philosophy, in which two fields of knowledge are articulated: studies on the body (fundamentally from philosophy and anthropology of the body) and the production around the teaching of philosophy. The fieldwork was performed between 2013 and 2014, the information processing was performed during 2015 and was publicly defended in 2016. The research was performed as a case study from a qualitative approach of ethnographic and interpretivist type. The relevance of the research itself is given by the need to embody the corporeal realm in teachers' education and specifically in philosophy teachers formation. The corporeal realm is understood as a constitutive aspect of subjectivity and, thus, of the way of existing and presenting of the subjects in the world. The goal of the research, even if it is framed as an exploratory study for not having a direct background on the field to inquire, refers to the need for integrating the work of the teaching object with himself, with its corporeality as an affective and affected body in the framework of the philosophical classroom.

Keywords: didactics of philosophy; bodies; body studies; teaching bodies.

\footnotetext{
This formation refers to an undergraduate formation which title is Medium Education Teacher with a Philosophy specialty, issued by the Uruguayan Educational Formation Council, Public Education National Administration. The formation here refers to training teachers for working, fundamentally, in medium school, which in Uruguay is commonly referred to as lyceum.
}

\section{Os corpos ensinantes na sala de aula de Filosofia}

\section{RESUMO}

Este texto corresponde aos principais resultados da pesquisa realizada com uma abordagem etnográfica com cinco estudantes de licenciatura em Filosofia* do Instituto de Profesores Artigas (IPA), da cidade de Montevidéu, Uruguai. Está delimitado no campo da didática da Filosofia, na qual são articulados dois campos de saberes: os estudos sobre o corpo (fundamentalmente a partir da filosofia e da antropologia do corpo) e as produções em torno do ensino da Filosofia. O trabalho de campo foi realizado entre 2013 e 2014; o processamento da informação foi realizado durante 2015 e o trabalho de conclusão de curso foi apresentado em 2016. A pesquisa é desenvolvida como estudo de caso, com uma abordagem qualitativa, de corte interpretativo e etnográfico. A rele- vância do estudo está na necessidade de incorporar o âmbito corporal ao campo da formação docente, em específico, à formação de docentes de Filosofia. O âmbito corporal é entendido como um aspecto constituinte da subjetividade e, por consequência, da forma de existir e apresentar-se dos sujeitos no mundo. A finalidade da pesquisa, embora esteja delimitada como estudo exploratório por não contar com antecedentes diretos sobre o campo em questão, refere à necessidade de integrar o trabalho do sujeito docente consigo mesmo, com sua própria corporeidade, enquanto corpos afetantes e afetados, no âmbito da sala de aula de Filosofia.

Palavras-chave: didática da Filosofia; corpos; estudos sobre o corpo; corpos ensinantes.

\footnotetext{
Esta formação se refere à graduação cujo título é professor de ensino médio, com especialização em Filosofia, emitido pelo Consejo de Formación en Educación de la Administración Nacional de Educación Pública de Uruguay. Tem o objetivo de formar docentes destinados a trabalhar fundamentalmente no ensino médio, o que tradicionalmente, no Uruguai, são denominados de "liceus".
} 


\section{Introducción}

El marco teórico de la investigación fue elaborado a partir de las producciones de varios autores que provienen fundamentalmente del área de la filosofía y su enseñanza, y de ámbitos relacionados con estudios específicos sobre el cuerpo y la corporeidad. En este sentido, se tomaron fundamentalmete como perspectiva teórica la fenomenología de Merleau Ponty; los estudios sobre género de Judith Butler y Marta Gómez, así como algunos aportes de Foucault; los estudios de Calmels $(2001,2013)$ del campo de la psicomotricidad; y desde la línea de la educación corporal que aborda el grupo de investigación coordinado por Luz Elena Gallo Cadavid de la Universidad de Antioquia en el campo de la educación física.

Desde el ámbito de la enseñanza de la filosofía, se tuvo en cuenta como referencia teórica lo elaborado por el grupo de trabajo de Didáctica de la Filosofía ${ }^{1}$ de IPA-CFE ${ }^{2}$, a partir de la perspectiva de Michel Foucault $(1979,2003)$, Michel Tozzi (1999) y Jorge Larrosa $(2000,2003,2006)$.

\section{Desarrollo del argumento del trabajo}

El problema central se circunscribe con relación a las siguientes preguntas:

- ¿Cómo se presentan los cuerpos enseñantes en el aula de filosofía?

- ¿Qué condiciones propician la enseñanza de la filosofía?

Estas preguntas suponen partir de algunas asunciones con relación a la enseñanza de la filosofía y la consideración de la corporeidad en el ámbito didáctico, así como la relevancia de esta en la enseñanza de la filosofía.

Si bien la corporeidad es ignorada en el análisis didáctico y fundamentalmente en el análisis didáctico en relación con el ámbito filosófico, esta genera condiciones para enseñar filosofía desde la modalidad desarrollada por el sujeto enseñante o cuerpo enseñante, en tanto constituye, a su vez, un aspecto de nuestra subjetividad.

Desde este lugar, se establece como objetivo general indagar e interpretar las prácticas corporales de los cuerpos enseñantes en el aula de filosofía, en tanto lugar de producción y creación.

Para poder profundizar en el análisis de las diferentes prácticas corporales, estas fueron pensadas en función de algunas focalizaciones, tales como la forma de habitar el espacio y de configurarlo por los cuerpos en el aula, la gestualidad

Grupo de trabajo conformado por las profesoras Isabel González Briz, Mónica Planchón y Janett Tourn.

2 IPA-CFE refiere a Instituto de Profesores Artigas del Consejo de Formación en Educación, instituciones públicas pertenecientes a la Administración Nacional de Educación Pública (ANEP) del Uruguay. 
del cuerpo enseñante, la cuestión de género en los cuerpos enseñantes, y la relación con la enseñanza de la filosofía de los aspectos indagados.

La categoría prácticas corporales fue articulada con la concepción de Judith Butler acerca de la conformación performativa del género (Butler, 1990). En este sentido, se entendieron las prácticas corporales como formas de decir del cuerpo, es decir, como formas de expresarse y de presentarse ante el mundo (Gallo, 2011, 2012, 2014).

A su vez, elaboré tres ejes de análisis que sirvieron para focalizar la observación y el procesamiento de los datos: el eje cuerpo espacio, el eje cuerpo expresión y el eje cuerpo género. Ninguna de estas referencias de análisis fue considerada de forma aislada, pues estas responden a una consideración de una existencia corporal generizada arraigada en el espacio. De todas maneras, la elaboración de tales ejes de análisis fue funcional a un criterio metodológico.

El eje cuerpo espacio se apoya fundamentalmente en la línea fenomenológica de Merleau-Pony (1975) y Wandelfels (2004). En este sentido, se considera fundamental la categoría habitar desde una doble acepción: desde el punto de vista antropológico - en tanto todos los seres humanos habitamos un espacio, un lugar-, así como desde una perspectiva más particular mediante la cual los cuerpos generan una implicancia afectiva con el espacio, por lo que, por ejemplo, la casa se transforma en nuestro hogar.

La categoría habitar refiere a una relación intrínseca entre los cuerpos y el espacio, mediante la cual el espacio se significa afectivamente y se crea a partir de los cuerpos. En este sentido, no habría una separación entre ambas acepciones de la categoría habitar, en tanto la afectividad sería una característica humana proyectada al espacio habitado. Sin embargo, también es posible pensar y observar que hay espacios que no son habitados sino ocupados, por lo cual me parece importante establecer una diferencia desde este punto de vista. Con respecto a este aspecto, la investigación refiere algunas conclusiones.

El eje cuerpo expresión se fundamenta también en las producciones de Merleau-Ponty bajo la idea del cuerpo como obra de arte, entendiéndolo en forma opuesta al cuerpo máquina de origen cartesiano. La expresión halla su asidero en la gestualidad, en la cual el significante y el significado no pueden deslindarse. Este eje se articula con la categoría gesto de Agamben (2001). Para este autor, el gesto es aquel movimiento diferenciado del puro hacer que descansa en la pura medialidad, es decir, en la afirmación de sí mismo y no en función de una finalidad ajena a él.

El eje cuerpo género se apoya bajo la línea de las investigaciones de Judith Butler (1990), para quien el género no refiere a ninguna entidad sustancial, sino 
que se constituye performativamente por repetición de actos mediante los cuales la norma se corporiza (Butler, 1990). En este sentido, Marta Gómez (2009) reflexiona sobre cómo el género se implica en el cuerpo de tal manera que se visibiliza a través de movimientos, gestos, maneras de caminar, sentarse, vestirse, etc.

La categoría cuerpo enseñante se estableció bajo la asunción antropológica de nuestra subjetividad encarnada, circunscribiendo la misma al contexto de aula y más específicamente al aula de filosofía.

Tomo, por tanto, una línea teórica conformada desde los autores anteriormente establecidos, línea que se asienta fundamentalmente en la perspectiva fenomenológica y la perspectiva performativa.

Cabe señalar que esta línea ha sido ampliamente tomada por la antropología del cuerpo que inauguró Mary Douglas (1921-2007) y que ha tenido un amplio desarrollo al integrar las concepciones de Merleau-Ponty desde la fenomenología, el estructuralismo, el posestructuralismo, las concepciones de Michel Foucault y Pierre Bourdieu. En este sentido, la antropología del cuerpo ha resaltado el carácter activo y transformador de las prácticas corporales. Por otro lado, se concibe el cuerpo como producto de adjudicaciones simbólicas y formaciones disciplinarias.

Cabe destacar en esta línea los trabajos de Silvia Citro $(2009,2010,2012)$ y el trabajo del grupo de estudio sobre cuerpo de la Universidad Nacional de la Plata desde un abordaje fenomenológico, como en Mora (2010). Desde otra línea de trabajo, a partir de las concepciones de Fernando Bárcena, Gilles Deleuze, Baruch Spinoza, cabe destacar los trabajos del Grupo de Investigación en Estudios en Educación Corporal coordinado por Luz Elena Gallo Cadavid (inició en la Universidad de Antioquia en el año 2003) desde una articulación más específica entre estudios sobre el cuerpo y la pedagogía, desde la educación corporal.

Por otra parte, cabe explicitar que se concibe el aula de filosofía como un lugar de recreación del saber filosófico y posibilitadora de condiciones para la transformación de los sujetos.

En este sentido, la categoría experiencia adquiere un lugar central, bajo el entendido de la necesidad de generar condiciones para que la misma acontezca (Foucault, 2003; Larrosa, 2003, 2006).

Dentro de estas condiciones, el cuerpo que somos adquiere un lugar de privilegio en tanto cuerpo afectante y afectado por otros y hacia otros. 
Por tanto, la indagación estuvo centrada en acercarme a la comprensión del cuerpo en el aula de filosofía, en tanto sujeto-cuerpo-enseñante generador de condiciones para enseñar filosofía.

En función de estas líneas teóricas, me interesa enunciar los aspectos que atraviesan la presente investigación y que, como tales, conforman la concepción sobre la subjetividad desde la cual trabajo. Esta concepción es compartida por numerosos investigadores y académicos actuales:

a. La subjetividad en tanto histórica y cultural.

b. La consideración del sujeto humano como sujeto encarnado generizado, corporeidad o sujeto-cuerpo y sus posibilidades expresivas.

C. El pensar en tanto pensar encarnado.

d. El vínculo como intercorporal.

e. El aspecto político del cuerpo, en tanto este también funciona como "superficie de inscripción" (Foucault, 1979) de las relaciones de poder.

f. Los cuerpos enseñantes en tanto sujetos encarnados, sujetos-cuerpos, afectados y afectantes.

Desde este lugar, las principales preguntas que atraviesan la investigación se circunscriben a continuación a partir de las dos preguntas centrales ya enunciadas.

Todas refieren al cuerpo enseñante, porque el interés específico es indagar sobre el cuerpo docente, específicamente sujeto-cuerpo docente de filosofía, en tanto este es quien propone un aula y desarrolla una forma de enseñar.

Las siguientes investigaciones deberían poder abordar los cuerpos de los estudiantes de enseñanza media en relación con la intercorporeidad, atravesada, en este caso, por el enseñar y aprender filosofía.

Entiendo que los procesos de enseñar y aprender son dos procesos muy complejos y diferentes que merecen una focalización diferenciada.

- ¿Qué decir de los cuerpos enseñantes en el aula de filosofía?

- ¿Cómo se presentan?

- ¿Qué mundo proponen a partir de su corporeidad y su modalidad de "ser cuerpo"?

- ¿Qué condiciones propician para enseñar filosofía? 
- ¿Qué relevancia tiene la forma de habitar el espacio para la enseñanza de la filosofía?

- ¿Qué construcciones de género se muestran en los cuerpos enseñantes?

- ¿Qué condiciones habilitan la expresividad observada en los cuerpos enseñantes para enseñar filosofía?

La investigación se enmarca bajo un estudio de caso desde una perspectiva cualitativa. Cada uno de los sujetos participantes de la investigación se constituyó en un caso y se utilizó el criterio de voluntad propia para participar en la investigación. El estudio de caso se caracterizó, entonces, por ser un estudio de caso instrumental (Stake, 1999), puesto que la finalidad de la investigación estuvo dada en la compresión de cómo se presentan los cuerpos enseñantes en el aula de filosofía y no específicamente en cada uno de los casos por la relevancia de estos. De todas maneras, la indagación se realizó de acuerdo con las características del estudio de caso cualitativo de forma profunda, a fin de comprender y no de medir ni de generalizar.

Se utilizó un abordaje etnográfico (Geertz, 2003) desde una perspectiva interpretativista (Álvarez, 2010). Dado que la cultura a indagar no se consideró extraña, sino como cultura compartida, se enmarcó como una autoobservación (Vallés, 1999) en tanto los casos son parte de mi propio contexto. La observación se constituyó en la principal estrategia. En la investigación participaron cinco estudiantes de Didáctica III $^{3}$ del Instituto de Profesores Artigas (IPA) de la ciudad de Montevideo, Uruguay. Se optó por invitar a estudiantes de Didáctica III por varias razones: en primer lugar, mi ámbito de trabajo y dedicación profesional se viene desarrollando en el ámbito de la formación docente desde hace varios años. Me interesa, entonces, indagar sobre las condiciones que hacen a la formación docente y específicamente a la formación de docentes de filosofía. Por otra parte, entendí que los estudiantes y las estudiantes de Didáctica III, por tener un grupo a cargo y por haber pasado ya por dos prácticas docentes anteriores ${ }^{4}$, han logrado un grado de autonomía importante que los posiciona como naturalmente predispuestos a participar en una investigación que propone focalizar en aspectos constituyentes de su

Didáctica III corresponde a una asignatura del $4 .^{\circ}$ año del profesorado, la cual consta de una parte teórica que se desarrolla como clase semanal y una parte práctica, la cual consiste en que el estudiante de profesorado se hace cargo a lo largo del año de un grupo de enseñanza media con el rol de profesor. La práctica implica la articulación de dos instituciones diferentes pertenecientes a ámbitos de la enseñanza diferentes. Por un lado, el Instituto de Formación Docente —en este caso el Instituto de Profesores Artigas (IPA) - perteneciente al ámbito del Consejo de Formación en Educación (CFE). Por otro lado, un liceo público perteneciente a la órbita del Consejo de Enseñanza Secundaria (CES). Ambos consejos son de la Administración de Educación Pública (ANEP).

4 Son prácticas docentes anteriores que corresponden a la carrera Didáctica I y Didáctica II, en este caso acompañado por un docente adscripto a cargo del grupo de enseñanza media. 
propia subjetividad, tal como el hecho de "ser cuerpo". A su vez, la función de mi propia trayectoria en el ámbito de la didáctica de la filosofía hace que la observación de clases no me sea ajena ni que los estudiantes del profesorado de Filosofía se sientan incómodos por ser observados.

Este último aspecto me parece muy relevante, en tanto se han generado las condiciones para compartir la intersubjetividad básica (Álvarez, 2010, p. 72) que, en la tradición fenomenológica, refiere al mundo de la vida, es decir, a los aspectos no medibles ni cuantificables de la existencia, fundamentalmente el ámbito de la vivencia. Esta intersubjetividad compartida habilita la comprensión y la interpretación, ya que comparto con los sujetos involucrados, además de un contexto histórico y social determinado —en forma más específica一, un mundo docente desde la propia disciplina y sobre todo desde el trayecto de formación.

Por ello, invité a los estudiantes de Didáctica III que cursaban en el 2013 a la presentación del proyecto de investigación a fin de dar conocer las finalidades de la investigación, así como su metodología y marco teórico. De este proceso resultaron integrados cuatro estudiantes, a quienes posteriormente se sumó un quinto estudiante. Los cinco estudiantes se corresponden con un perfil socioeconómico de clase media. Los estudiantes, tres chicas y dos varones, tenían entre veinte y treinta años.

Los grupos a cargo de los estudiantes corresponden a grupos del segundo ciclo de enseñanza media (es decir, cuarto, quinto y sexto), es decir, los últimos años de formación correspondientes a la enseñanza media. La distribución de las prácticas docentes, esto es, del grupo a cargo de cada uno de ellos resultó en:

- Una estudiante mujer a cargo de un grupo de quinto año de enseñanza media en liceo extraedad ${ }^{5}$ diurno en el centro de la ciudad.

- Una estudiante mujer a cargo de un grupo de quinto año de enseñanza media en liceo extraedad nocturno ubicado al este de la ciudad.

- Una estudiante mujer a cargo de un grupo de sexto año de enseñanza media en un liceo extraedad nocturno ubicado en la zona oeste de la cuidad.

- Un estudiante varón a cargo de un grupo de cuarto año de enseñanza media diurno en liceo en la zona oeste más periférica de la cuidad.

- Un estudiante varón a cargo de un grupo de sexto año de enseñanza media diurno en liceo en la zona este de la cuidad.

Extraedad se refiere a adultos, en tanto que la enseñanza media está dirigida en principio a quienes egresan de primaria, es decir, desde los doce años hasta los dieciocho años de edad. Luego de esa edad se consideran como extraedad. 
Se acordó con ellos la filmación de una de sus clases de dos horas de trabajo, así como la realización de un grupo de discusión para realizarlo después de las filmaciones. Opté por realizar las filmaciones de clase luego de que los estudiantes hubiesen rendido su examen de didáctica-práctica docente y de que se hubiesen tramitado los permisos correspondientes ${ }^{6}$. Una vez realizadas cada una de las filmaciones de clase, invité a los estudiantes participantes de la investigación a un grupo de discusión. Este estuvo precedido por un trabajo corporal a manera de sensibilizar la discusión para luego presentarles una serie de textos filosóficos y no filosóficos cuya temática estuviese en torno a la categoría cuerpo.

La finalidad del grupo de discusión estuvo dada en poder acercarme a sus creencias y convicciones en torno a la categoría cuerpo y su posible relevancia en el ámbito de la enseñanza de la filosofía. Con respecto al procesamiento de datos, profundicé en cada caso por separado en primera instancia, para luego acceder a las reflexiones finales. Cada uno de los casos, siguiendo a Simons (2011) fueron procesados de acuerdo con tres fases: descripción, análisis e interpretación. Por medio de la descripción intenté mostrar lo observado en la clase presenciada y filmada, atendiendo a las siguientes categorías:

Espacio físico: estado general del salón, ubicación de las puertas, ventanas, iluminación, estado de las paredes, ubicación de muebles tales como pizarrón, escritorio y bancos.

Ubicación del estudiante practicante $(E P)^{7}$, ubicación de los estudiantes $(E)$; configuración del espacio de acuerdo con estas ubicaciones:

- Desplazamientos del EP y posturas corporales.

- Gestos y expresiones del EP.

- Cantidad de E, sexos, edades aproximadas.

- Relacionamiento de los E con el EP.

- Inicio de la clase, clima de la clase.

- Otros en función de cada uno de los casos.

$6 \quad$ Estos permisos se piden ante el Consejo de Educación Secundaria para ingresar a las aulas seleccionadas y realizar filmaciones, ante las direcciones de los diferentes liceos y los estudiantes de secundaria. En el caso de los grupos extraedad, estos fueron gestionados por los estudiantes de Filosofía y en el caso de los menores se realizó por medio de las direcciones de los liceos dirigido a los padres de los estudiantes.

7 EP refiere a estudiante practicante, es decir, el estudiante del profesorado de Filosofía, mientras que $\mathrm{E}$ refiere a los estudiantes de enseñanza media. 
A partir de la descripción, opté por analizar y a la misma vez interpretar la descripción lograda - sin desentenderme de la filmación y las anotaciones realizadas al observar cada una de las clases- a partir de algunos ejes teóricos que me sirvieron para organizar lo descrito y comenzar a encontrar sentidos. Dado el marco teórico adoptado y construido desde las lecturas realizadas y el acercamiento a la obra del filósofo Maurice Merleau-Ponty, se postularon las siguientes categorías de análisis: cuerpo-espacio; cuerpo expresivo; y posteriormente incluí cuerpo-género.

El análisis y la interpretación de cada uno de los casos se fue enriqueciendo a partir de ir encontrando otros aspectos en la modalidad corporal observada de los EP, lo que implicó en cada caso integrar reflexiones puntuales. En cada caso me detuve a analizar e interpretar el aspecto corporal, que a mi juicio diera el rasgo más distintivo del EP y su clase. Podría decir que, en este sentido, esta forma de procesar los datos se acercó al uso de la categoría emergente ${ }^{8}$ usada de forma similar a como la incluye la psicología social desde la línea desarrollada por Pichón (1999). A partir del procesamiento de cada caso en forma independiente, se procesaron todas las categorías y ejes de análisis en función de las preguntas y objetivos de la investigación para acceder a las conclusiones finales.

Así, la primera línea de reflexión final estuvo dada a partir de la categoría habitar (Merleau Ponty, 2008) referida a la forma de presentarse los cuerpos docentes en el aula en relación con el espacio. En el caso de dos de las estudiantes practicantes (EP3 Y EP4) este aspecto se observa a través de la apropiación del espacio que realizan junto con sus estudiantes. Esta apropiación refiere a una intervención espacial a partir de la disposición en círculo en el cual se configura un orden espacial distinto al dispuesto institucionalmente. La disposición institucional tradicional responde a una determinada concepción de la enseñanza, en la cual hay un lugar ya establecido para el docente poseedor del saber y un lugar para los alumnos enfrentados al docente, dispuestos a recibir el saber 9 . En esta los alumnos no se miran entre sí, lo cual no propicia el diálogo entre ellos, sino que se dispone a una comunicación solo con el docente. A su vez, esta disposición se corresponde con lo que es posible denominar "una concepción homogénea del espacio". En este sentido, este es considerado independiente de las corporeidades y pensado, entonces, desde un orden institucional en forma abstracta, pero con un orden previamente instalado (lugares ya establecidos entre docente y alumnos).

8 El uso de tal categoría en la psicología social de cuño pichoniano puede traducirse como lo que muestra, explicita. Los emergentes o el emergente deben ser develados por un proceso de interpretación de lo que sucede en la dinámica grupal observada. Obviamente también se habla aquí de interpretación por parte del analista, psicólogo social.

$9 \quad$ Enseñanza denominada bancaria desde el planteamiento de Paulo Freire. 
Las dos estudiantes, junto con sus alumnos, intervienen el espacio y generan un grupo pequeño en el cual los cuerpos son fácilmente discernibles y en el que las relaciones y los vínculos se desarrollan en una mayor intimidad. El grupo pequeño hace referencia a un criterio cuantitativo, en tanto se componen de diez a doce alumnos en ambos casos, pero que se articula, a su vez, con una nueva configuración espacial que rompe la configuración tradicional adjudicable al espacio homogéneo de las instituciones educativas pensadas a partir de la concepción tradicional de enseñanza, que no es más que una concepción bancaria.

Por lo tanto, en los casos de las dos estudiantes se habita el espacio y no solo lo ocupan. Hay una apropiación de este que posibilita una relación intercorporal diferente que habilita a la grupalidad ${ }^{10}$, es decir, la generación de vínculos entre los integrantes del grupo que construye una cierta identidad grupal y no a una mera sumatoria de individuos. La posibilidad de habitar los espacios, es decir, de apropiarse de estos, también se establece integrando las condiciones objetivas: los dos grupos están compuestos por muy pocos estudiantes. Es posible acceder, entonces, a una relación habitar-grupo pequeño.

Por otra parte, las condiciones físicas de los salones en los que habitan estos dos grupos a cargo de las dos estudiantes EP3 y EP4 se corresponden con condiciones que se traducen en deterioro de los espacios o en la no apropiación de estos: salones en los cuales se observan paredes gastadas o pintadas, pero sin huellas de identidad. Este aspecto hace más valorable la apropiación del espacio que realizan estas dos estudiantes practicantes, porque adaptan el espacio a su propia dinámica.

A su vez, la construcción de género hegemónica, entendiendo esta como la que corresponde a la binariedad mujer-hombre, atraviesa esta apropiación del espacio. Así, el espacio generado por las dos estudiantes responde a lo que podemos denominar espacio femenino, en tanto puede ser pensado como un espacio intimista en el cual los cuerpos de los habitantes se disponen en forma circular y con una distancia física entre sí muy pequeña. En el caso de la estudiante EP3 se reafirma este aspecto a través del mate ${ }^{11}$ que sostiene por momentos algún juego de poder con un estudiante. Es posible, entonces, realizar otra relación entre espacio habitable, intervenido (grupo pequeño) y espacio que denominaré femenino. Con respecto a esto, es necesario preguntarse: ¿la intervención, la transformación, el establecimiento de condiciones distintas a las hegemónicas será, entonces, llevada adelante por mujeres docentes desde su impronta de género?

Refiere a la idea de grupo de E. Pichón Riviere y que es retomada por ejemplo por Marta Souto (1993).

11 El mate es una infusión que se realiza con la yerba mate, característica de Uruguay, Argentina, Paraguay y del sur del Brasil. 
Esta sospecha es reforzada al pensar en uno de los estudiantes practicantes, el EP2, quien propicia una forma distinta de volver habitable el espacio. Así, esta es realizada a partir de su desplazamiento de manera instrumental, mediante el cual establece una relación entre los cuerpos de los estudiantes. Esta forma de habitar el espacio está centrada en el cuerpo del EP. En este sentido, el estudiante les propone a sus alumnos una tarea por subgrupos dentro del mismo salón, salón que responde a la disposición tradicional bancaria. Los estudiantes se agrupan sin desplazarse demasiado, conformando pequeños subgrupos. El EP2 recorre ágilmente los subgrupos generando con su propio cuerpo en desplazamiento un entretejido entre los diferentes subgrupos. En este sentido, es posible afirmar que la centralidad está dada en el cuerpo del estudiante practicante y no en una intervención que genere una transformación espacial dirigida haca la conformación de la grupalidad y, por tanto, una transformación en la disposición de los cuerpos de los estudiantes.

No deja de ser interesante remarcar, además, que esta forma de intervenir es realizada desde lo instrumental, desde la búsqueda del fin, del objetivo. Es interesante pensar que esta apropiación del espacio también se da desde un cuerpo generizado por sus movimientos, su decisión, su seguridad, su forma resuelta de apropiarse del espacio, sus movimientos rápidos y rectos, su expresividad, su expresión de seriedad en el rostro y por el acento del desplazamiento puesto en la finalidad. Esto quiere decir que se da una intervención espacial desde una generización masculina que sigue siendo central en su corporeidad en la configuración del aula, lo que establece un claro dominio desde su presencia corporal. Por ello, cabe pensar en un espacio habitable, intervenido, dominado, espacio que llamaré masculino por las características asignables desde una cierta hegemonía del estereotipo masculino.

De todas maneras, es necesario establecer que existe en el EP2 una apuesta al cambio, pero que se resuelve hegemónicamente a través de la acción del docente varón: intervención del espacio desde la masculinidad entendida como construcción de género hegemónica.

En los otros dos casos, una EP1 y un EP5, los cuerpos se adaptan al espacio institucionalizado, proyectado desde un espacio homogéneo característico de las instituciones educativas. También en estos casos se dan condiciones objetivas que hacen difícil apropiarse de manera distinta del espacio: los dos grupos de estudiantes son muy grandes y el salón está repleto de bancos.

Es posible, entonces, realizar una relación que tiende a mantenerse entre la no intervención del espacio, la adaptación a la lógica institucional y el espacio repleto de objetos, espacio ocupado. 
La construcción de género hegemónica, esto es, la binariedad de género en masculino/femenino, atraviesa las corporeidades de los EP. Esto se observa tanto en la manera en que habitan el espacio, así como en su expresividad de la siguiente manera: la forma intimista de habitar el espacio en las dos estudiantes practicantes referidas se espera en la caracterización mujer, así como en una mínima apropiación del mismo por parte de la otra estudiante practicante (EP1).

Esta construcción de género está atravesado por la dulzura en la voz, la sonrisa que fácilmente aparece en el rostro de EP1 y que también caracteriza a la forma de habitar de la EP4 con algunas diferencias, pues EP4 conforma un estereotipo femenino más centrado en modalidades en las cuales su imagen parece ser un aspecto de su interés, así como en posturas claramente asignables a la construcción hegemónica del género femenino como cruzar las piernas. Sin embargo, la seriedad caracteriza la expresión del rostro de la EP3 quien, si bien construye junto con sus estudiantes un espacio pequeño, íntimo, sostiene con su mirada el vínculo intercorporal en un rostro en el cual la sonrisa no está presente.

En la forma de apropiarse del espacio y de habitarlo por parte de los estudiantes practicantes varones también es posible rastrear construcciones de género asignables a la caracterización hombre con diferencias en ambos. En tanto para uno de ellos el desplazamiento se vuelve su forma de presentarse y su forma de mostrar el poder de su cuerpo, para el otro (EP5) su modalidad corporal desarrollada a través de sus movimientos rectos, ágiles, así como sus posturas sobre el escritorio, también dan cuenta de una masculinidad expresable que establece una propuesta de clase en la cual su dominio se da fundamentalmente a través de la voz.

El EP5 se ubica frente a sus alumnos, es decir, ocupa el lugar tradicional asignable al docente y de acuerdo con la espacialidad establecida por las instituciones educativas, pero lo hace fundamentalmente sentado encima del escritorio. Por ello, si bien es posible establecer cierta transgresión corporal, sus movimientos, el uso de su voz, lo ubican desde una hegemonía de género masculino. Por otra parte, la expresión de seriedad caracteriza a ambos (EP2 y EP5).

Los matices y diferencias de los cinco practicantes muestran construcciones de género hegemónicas que, sin embargo, no parecen ser registradas a nivel discursivo, tal como establecen en el grupo de discusión realizado.

En cuanto a la presencia de una lógica institucional que establece los lugares de acuerdo con una espacialidad concebida desde la homogeneidad —espacio homogéneo tal como fue caracterizado antes- tiende a mantenerse en todos los casos observados. Así, el aquí, en tanto lugar de la enunciación, tiende a no cambiar, pues en tres de las cinco aulas observadas el lugar del docente de 
espaldas al pizarrón y de frente a sus alumnos se mantiene, lo que responde a la lógica homogénea prevista a nivel institucional. Por lo tanto, este aquí configura las coordenadas espaciales en tanto punto cero desde el cual se emite la enunciación. El adelante y el atrás tienden a permanecer, exceptuando los dos casos en los cuales se configura el grupo pequeño.

De todas maneras, no deja de ser interesante establecer que la EP1, que no logra apropiarse del espacio, queda casi aprisionada por su propio cuerpo al cual considera un lastre, algo que desconoce ${ }^{12}$. Se da una sola vez la posibilidad de cruzar el espacio sin una finalidad determinada, como si estuviera intentado descubrir otros lugares, otras perspectivas, experimentarse a sí misma en relación con el espacio y, por tanto - aunque lo niegue-, experimentarse en relación con su cuerpo, con su corporeidad. Parafraseando a Masschelein (2006), es como si fuese posible decir que hay un intento de experimentar la novedad, la búsqueda sin buscar nada en especial.

Es posible, entonces, establecer un quiebre entre lo observable en las prácticas corporales y el discurso. Este aspecto me parece sumamente interesante, pues de alguna manera evidencia la relevancia de lo corporal, por ejemplo, desde el punto de vista de las inscripciones y disciplinamientos (Foucault, 1979), sin que esto sea concientizado por los sujetos. Es más, se evidencia la complejidad de una razón encarnada que se niega a regirse por el principio de no contradicción. La racionalidad hegemónica occidental (Morin, 2001) logra verse quebrada por la presentación de los cuerpos enseñantes. Esto quiere decir que, más allá de las enunciaciones de los sujetos, la razón encarnada logra explicitarse en la corporeidad generizada. Si bien hay una mínima apropiación del espacio por parte de esta estudiante (EP1), cuando por una sola vez camina hacia el fondo del salón, se presenta un quiebre de su propia concepción y registro del lugar del cuerpo.

En cuanto a la gestualidad pensada desde el eje cuerpo obra de arte:

La voz, si bien está presente en todos los EP, logra su clara relevancia en el EP5 a través de la lectura en voz alta. Esta voz se posiciona expresivamente y domina el espacio, aún en los momentos de ignorancia que se muestra en la expresión de las corporeidades de los estudiantes con un cierto tedio. De todas maneras, esta voz se plantea desde una cierta puesta en escena para los estu-

12 Me refiero en este caso a su propia declaración en grupo de discusión cuando dice: “(...) debo decir que me considero cartesiana o incluso platónica. El cuerpo es un lastre. No tengo casi conciencia de mi cuerpo, en verdad no sé si me siento un cuerpo, no siento mi cuerpo, en verdad no sé bien como es en realidad, como se ve. Yo me siento una mente, y puedo dar cuenta de cada pensamiento, estado de ánimo, analizar lo que pienso, seguir la cadena causal de pensamientos que me trajeron a lo que estoy pensando ahora, analizar incluso lo que por ejemplo se dijo en clase, todo eso queda muy patente, pero no logro recordar a veces cómo son los estudiantes, no los reconozco, no recuerdo dónde se sientan (...)" (Tourn, 2016). 
diantes o para la filmación por momentos, poniendo en movimiento un efecto de seducción. Es posible, entonces, establecer una relación entre la voz y dominio del espacio desde una generización masculina.

En el caso de la EP1, la voz que se conjuga con gestos expresivos, tales como palmas hacia arriba y mirada en una dirección lejana, muestran un pensar activo que invita claramente a la reflexión. En este sentido, es interesante plantear cómo la enseñanza de la filosofía en tanto enseñanza que promueve el pensar, la autoría, la creatividad, puede quebrar la construcción hegemónica de género, al menos se observa este aspecto en la EP1 cuando logra momentos reflexivos claramente visibles corporalmente. Este aspecto me lleva a establecer una relación entre enseñar filosofía y prácticas de libertad, en tanto la enseñanza de la filosofía se comprende como una actividad que propicia la autoría del pensar tanto del docente como del alumno. Asimismo, la creatividad encarnada en el pensar logra visiblemente que esta estudiante genere transformaciones en su corporeidad, las cuales se manifiestan a partir de gestos que pueden pasar desapercibidos, pero que están mostrando un pensar activo. Sobre todo, se acentúa que esta estudiante es quien declaraba no ser consciente de su cuerpo.

En todos los estudiantes practicantes, la mirada y el encuentro con la mirada de los estudiantes alumnos está presente. Pero sobre todo interesa acentuar la relación cara a cara, relación ética por excelencia (Levinas, 2002) que caracteriza a una de las aulas observadas (EP3). Esta estudiante sostiene un vínculo intercorporal que se desarrolla bajo la comodidad y el diálogo entre los integrantes del pequeño grupo, que no excluye, sin embargo, algún momento de tensión. Este pequeño grupo, constituido bajo la relación cara a cara, habilita el diálogo filosófico. En este diálogo los sujetos se muestran, explicitan sus creencias y argumentos, y dan la posibilidad para que el sujeto se transforme. Este diálogo lo debemos entender como discusión filosófica (Berttolini, 2009), en tanto que los sujetos se contaminan ${ }^{13}$ entre sí alterando su subjetividad. Es posible establecer una relación entre el cara a cara, el diálogo filosófico y la transformación de sí, en tanto el diálogo promueve que los sujetos no permanezcan de la misma manera que antes.

La lectura también ocupa un lugar importante en dos de las aulas observadas, como en el caso de EP4 y EP5, con lo que se configura lo que podemos llamar el cuerpo lector. Estos cuerpos lectores, atravesados por construcciones de género, cuerpos atentos, tensos, sostienen el silencio de la lectura. Ambos estudiantes practicantes sostienen tiempos dedicados a la lectura con una disposición corporal dedicada a ello: el estudiante EP5 lo hace desde

13 Contaminar en el sentido que lo plantea Berttolini (2009), en tanto los sujetos logran quebrar su aislamiento mediante la discusión, recuperan un espacio público de discusión, espacio común en el cual nadie permanece igual si la discusión es sincera y argumentada. 
una posición de dominio, sentado sobre el escritorio desde un plano más alto que los estudiantes, leyendo él mismo con mucha seguridad y seducción. La estudiante EP4 no lee, pero atiende a la lectura realizada por un alumno desde un banco en el cual su corporeidad se encuentra atravesada genéricamente con las piernas cruzadas y el tronco inclinado hacia adelante. Esto quiere decir que él (EP5) lee seductoramente, mientras que ella (EP4) escucha la lectura cuasi flexionada sobre sí misma. Desde sus corporeidades, podemos traducir sobre él una apertura al mundo y dominio, y sobre ella un recogimiento sobre sí misma desde un cruce de piernas, en la cual la escucha ocupa un lugar importante.

El silencio que acompaña a la lectura, pensado como aspecto emergente, destacable, que dice cosas — paradójicamente-, se hace presente desde dos significaciones distintas. Por un lado, un silencio que sostiene la lectura en voz alta del estudiante practicante (EP5) y la lectura de los estudiantes alumnos en el aula de la estudiante practicante (EP4) también en voz alta, que llena el espacio y sostiene el cuerpo lector, tenso, concentrado, con gesto filosófico y reflexivo. Esto se da en ambos casos. Por otro lado, un silencio cargado de espera es observable en la estudiante practicante (EP4) y hasta de cierta ansiedad por escuchar la respuesta correcta o, al menos, una respuesta. Este silencio está acompañado de gestos que muestran cierta incomodidad, tal como morderse el labio. Este silencio, silencio cero - por oposición al silencio reflexivo- no ofrece condiciones para la experiencia filosófica ${ }^{14}$ porque espera una respuesta determinada. El silencio expectante, cargado de espera, no puede escuchar lo nuevo porque quiere ser llenado de lo ya construido a manera de la pieza que falta.

\section{Conclusiones. En torno a la relación con el enseñar filosofía}

¿Qué condiciones propician estos cuerpos, tal como han sido observados, para la enseñanza de la filosofía? ¿Qué posibilidades ofrecen habitar la espacialidad, la expresividad, las diferentes construcciones de género para enseñar filosofía? ¿Es posible acceder a un gesto filosófico?

La concepción acerca de enseñar filosofía se basa fundamentalmente en la idea de que el aula de filosofía debe propiciar espacios mediante los cuales la disciplina filosófica esté presente, para producir condiciones que habiliten la transformación de sí.

14 Experiencia filosófica remite a una diferencia que realiza Foucault (2003) entre libros experiencia y libros verdad. Los libros experiencia tienen la finalidad de transformar al propio autor, en tanto los libros verdad quieren demostrar una verdad previamente establecida. Desde este lugar, el aula de filosofía se concibe desde la posibilidad de generar las condiciones para la experiencia, es decir, para que los sujetos puedan transformarse. Esta categoría es ampliamente tomada por varios autores entre ellos Jorge Larrosa (2006). 
- ¿Qué condiciones habilitan los cuerpos enseñantes para la transformación de sí?

- ¿Es posible acceder a este plano de la subjetividad?

Entiendo que es posible acceder a algunas conclusiones en función del marco teórico y metodológico señalado, que deben ser pensadas como condiciones habilitantes para la enseñanza de la filosofía. En este sentido, es posible señalar lo siguiente:

El habitar mediante la construcción de grupos pequeños genera un espacio que propicia el diálogo, en tanto este necesita de la exposición de los sujetos en un clima de confianza, lugar que se construye mediante cuerpos que se vuelven discernibles y que se exponen generosamente al otro. El diálogo filosófico entendido como discusión filosófica "sacude el pensamiento, agita, interpelando, los saberes instituidos (conocimientos, prácticas, valores), no les permite cristalizarse" (Berttolini, 2009, p. 5). La relación cara a cara que dispone la grupalidad posibilita las condiciones para la remoción de saberes, creencias y prácticas. Es un cuerpo que se sacude y posibilita la transformación de aquello que parece incambiable. Se generan condiciones para la experiencia, es decir, para que algo pase en términos de Larrosa $(2000,2003,2006)$ y Foucault (1979, 2003), para que algo me golpee y me movilice.

El espacio íntimo que tiende a conformarse, el espacio femenino, genera condiciones para un pensar no hegemónico. Así, se toma la historia de la filosofía como una historia en la cual las mujeres han estado, con excepciones, excluidas (Tourn, 2016, pp. 126-127).

Si el pensar se realiza desde sujetos concretos, desde cuerpos concretos, entonces el aquí configurado también da forma a este pensar. Por ello, se vuelve muy relevante la corporeidad que piensa, y esta no puede ser despojada de su espacialidad y de su forma de habitar el espacio. Se piensa desde esta forma concreta de habitar y de interactuar con otros. Se piensa desde la transgresión o desde la adaptación a las condiciones hegemónicas; se piensa desde el dominio, desde la pasividad o desde la posibilidad de transformarse también a sí mismo.

Por ello, las posibilidades de transgredir el espacio institucional en tanto espacio homogéneo habilita la transgresión del pensamiento y, por tanto, la creatividad. Estos aspectos son fundamentales en la filosofía, en tanto esta es entendida como recreación de la disciplina y no repetición, donde el sujeto es interpelado por el pensar. La posibilidad de transgresión de los espacios genera condiciones para la creación, para recrear el pensar e incluso para generar condiciones de autoría del pensar. 
La expresión, la gestualidad que toma cuerpo en la pura medialidad que lo habita, que tiende a la expansión del sujeto, permite su propia transformación. La potencialidad expresiva en sí misma, sin volverse un medio al servicio de una finalidad meramente instrumental, da cuenta del sujeto en el espacio, en su cuerpo, volcado a la sensibilidad, a la conciencia corporal y al encuentro con la experiencia. Esto quiere decir que la gestualidad posiciona al sujeto enseñante en un presente e intensifica las posibilidades del encuentro con lo que puede conmover, afectar y, en definitiva, transformar. Parafraseando a Jorge Larrosa (2003), es una invitación a detener el tiempo y acentuar la sensibilidad ante y con los otros.

Ejemplo de estos gestos, que me animaría a establecer como gestos filosóficos, son el caminar sin objetivo determinado, palmas que se abren al mismo tiempo que se establecen preguntas interpelantes, relaciones cara a cara, miradas expectantes ante lo que el otro va a decir, tensión del cuerpo lector, silencios que sostienen al cuerpo lector.

Las significaciones sobre cómo se presentan los cuerpos pensantes, las cuales habilitan a la enseñanza de la filosofía en tanto esta promueve las condiciones para la transformación de los sujetos, se circunscriben a un cuerpo que esencialmente goza de su estar y de su pensar, lo que posibilita esencialmente el encuentro con el otro, aun en cuerpos que muestran rastros de los disciplinamientos y de construcciones de género hegemónicas. Incluso con modalidades distintas, dos de las EP manifiestan verbalmente la ausencia, la falta, el no saber sobre el registro de su cuerpo en el aula, como si este fuera un lastre, o el no poder trabajar desde el cuerpo. Por otra parte, el EP2 da cuenta de la pesantez de su cuerpo al concientizarse que la clase falló.

En estas verbalizaciones hay algo del orden de la experiencia, hay un registro de que algo está sucediendo, paradójicamente, en relación con una ausencia, a lo que no está, pero que se vive como algo que de alguna manera impacta, por ejemplo, a través de la mirada de los otros sobre sí, y que remueve la experiencia de "ser cuerpo" (Tourn, 2016, p. 128).

La experiencia de realizar esta investigación me ubicó claramente en la afirmación de la relevancia de lo vincular desde la intercorporeidad y, por ende, en la necesidad de incorporar en la formación de los docentes un espacio dedicado al trabajo con su propia corporeidad. Creo que los discursos sobre la educación han avanzado en criticidad, pero las prácticas se siguen moviendo en el afianzamiento del dualismo, en tanto el sujeto sigue siendo dividido en su escolarización: se escinde el pensar de su corporeidad. 
En el caso de la enseñanza de la filosofía, este aspecto se profundiza por las mismas características de la disciplina como campo de saber. Pero si somos cuerpos, es claro que el pensar parte del cuerpo y que la incorporación de la corporeidad de una forma más consciente puede profundizar también la necesaria creatividad filosófica.

Por otra parte, a nivel personal, la investigación me aportó especialmente una mirada más generizada hacia mí misma. Este aspecto no dejó de sorprenderme, puesto que me enfrentó con mi propia subjetividad. Este aspecto me parece muy valorable como resultado de una investigación en la cual mi propia subjetividad estuvo implicada desde el inicio como el principal instrumento de investigación.

\section{Agradecimientos}

El presente artículo recoge las principales líneas y resultados de la investigación realizada como tesis de maestría en Enseñanza Media, cursada y aprobada en el Instituto Universitario de Economía Humana (Claeh), Montevideo, Uruguay. Dicha investigación lleva por título Cuerpo y enseñanza de la filosofía, realizada entre el 2015 y el 2016.

\section{Referencias}

Agamben, G. (2001). Medios sin fin: Notas sobre la política. Pre-textos.

Álvarez, E. (2010). Tras la exploración de lo cualitativo y singular, fenomenología, hermenéutica y más allá. En J. Rasner (coord.), De la epistemología a la metodología y viceversa: Una aproximación a la investigación en ciencias humanas y sociales (pp. 69-138). Unidad Central de Educación Permanente de la Universidad de la República de Uruguay.

Berttolini, M. (2009). La educación filosófica para la reconstrucción de las subjetividades. https://docs. google.com/viewer?a=v\&pid=sites\&srcid=ZGVmYXVsdGRvbWFpbnxkaWRhY3RpY2FmaWxvc 29maWNhfGd4OjO5MjE4NTEzOTA4OGVIMmI

Butler, J. (1990). Actos performativos y constitución del género: Un ensayo sobre fenomenología y teoría feminista. En S. Ellen Case (ed.), Performing Feminisms: Feminist Critical Theory and Theatre (pp. 270-282). Baltimore University Press.

Calmels, D. (2001). Espacio habitado en la vida cotidiana y la práctica psicomotriz. Novedades Educativas.

Calmels, D. (2013). Fugas: El fin del cuerpo en los comienzos del milenio. Biblos.

Citro, S. (2009). Cuerpos significantes: Travesías de una etnografía dialéctica. Biblos.

Citro, S. (2010). Cuerpos plurales: Antropología de y desde los cuerpos. Biblios.

Citro, S. (2012). Cuerpos en movimientos: Antropología de y desde las danzas. Biblos.

Foucault, M. (1979). Microfísica del poder. Las Ediciones de La Piqueta.

Foucault, M. (2003). El yo minimalista y otras conversaciones. La Marca. 
Gallo, L. (2011). La educación corporal bajo la figura del acontecimiento. Revista Educación Física y deporte, 30(2), 505-513. https://revistas.udea.edu.co/index.php/educacionfisicaydeporte/article/ view/11310

Gallo, L. (2012). Las prácticas corporales en la educación corporal. Revista Brasileira de Ciências do Esporte, 34(4), 825-843. https://cev.org.br/biblioteca/praticas-corporais-educacao-corporal

Gallo, L. (2014). Expresiones de lo sensible: lecturas en clave pedagógica. Eduçâo e Pesquisa: Revista da Faculdade de Educaçâo da USP, 40(1), 197- 214. https://doi.org/10.1590/S1517-97022013005000027

Geertz, C. (2003). La interpretación de las culturas. Gedisa

Gómez, M. (2009). El género en el cuerpo. Avá, 15, 289-306. http://www.ava.unam.edu.ar/images/15/ pdf/ava15_15_gomez.pdf

Larrosa, J. (2000). Pedagogía profana: Estudios sobre lenguaje, subjetividad y formación. Novedades Educativas.

Larrosa, J. (2003). Entre las lenguas: Lenguaje y educación después de Babel. Laertes.

Larrosa, J. (2006). Sobre la experiencia. Aloma: Revista de Psicología, Ciències de l'Educació i de l'Esport, $19,87-112$.

Levinas, E. (2002). Totalidad e infinito. Sígueme.

Masschelein, J. (2006). Educar la mirada. La necesidad de una pedagogía pobre. En l. Dussel y D. Gutiérrez (comps.), Educar la mirada. Políticas y pedagogías de la imagen (pp. 295-304). Manantial, Flacso, Osde.

Merleau-Ponty, M. (1975). Fenomenología de la percepción. Gallimard.

Merleau-Ponty, M. (2008). El mundo de la percepción. Fondo de Cultura Económica.

Mora, A. S. (2010). Entre las zapatillas de punta y los pies descalzos. Incorporación, experiencia corporizada y agencia en el aprendizaje de danza clásica y contemporánea. En S. Citro (coord.), Cuerpos plurales. Antropología de y desde los cuerpos (pp. 219 238). Biblos.

Morin, E. (2001). Introducción al pensamiento complejo. Gedisa.

Nietzsche, F. (1990). La gaya ciencia. Yericó. Madrid España

Pichón, E. (1999). El proceso grupal: del psicoanálisis a la psicología social (1). Nueva Visión.

Simons, H. (2011). El estudio de caso: teoría y práctica. Ediciones Morata.

Souto, M. (1993). Hacia una didáctica de lo grupal. Miño y Dávila editores.

Stake, R. (1999). Investigación con estudio de casos. Ediciones Morata.

Tourn, J. (2016). Cuerpo y enseñanza de la filosofía [tesis de maestría, Instituto Universitario, Centro Latinoamericano de Economía Humana, Uruguay]. Recursos educativos, Uruguay Educa. https:// uruguayeduca.anep.edu.uy/sites/default/files/2018-04/Janett\%20Tourn\%20Maestr\%C3\%ADa\%20 en\%20Ense\%C3\%B1anza\%20Media\%20.pdf

Tozzi, M. M. (1999). Pensar por sí mismo. Introducción a la pedagogía de la filosofía. Popular.

Universidad de Antioquía. (s.f.). Grupos de investigación. Consultado el 28 de septiembre de 2019. http:// www.udea.edu.co/wps/portal/udea/web/inicio/institucional/unidades-academicas/institutos/ educacion-fisica-deportes/investigacion 
Valles, M. (1999). Técnicas cualitativas de investigación social: Reflexión metodológica y práctica profesional. Síntesis.

Wandelfels, B. (2004). Habitar corporalmente en el espacio. Daimon Revista Internacional de Filosofía, 32, 21-37. http://revistas.um.es/daimon/article/download/15221/14681 